\title{
Mediatisierte Lebensführung und ihr Wandel durch Alltagsumbrüche
}

\author{
Zur Relevanz von Übergangsphasen in der Rezeptionsforschung
}

Stephan Niemand ${ }^{*}$

In dem Artikel wird das Konzept der mediatisierten Lebensführung entfaltet, das sich speziell dazu eignet, um den Wandel des Medienhandelns im Zuge von Alltagsumbrüchen wie Trennungen, Elternschaft oder Wohnungswechsel zu analysieren. Eine zentrale Besonderheit des Konzepts ist, dass es den Alltag in die Strukturdimensionen zeitlich, räumlich, inhaltlich, sozial, sinnbezogen, materiell, emotional und körperlich aufspannt. So können Veränderungen in der Alltagsstruktur zielgenau und systematisch mit einem Wandel des Medienhandels in Beziebung gesetzt werden. Die empirische Reichweite des Konzepts wird anhand der Befunde einer qualitativen Panelstudie veranschaulicht. Es wird gezeigt, inwiefern alltagsspezifische Antriebfaktoren, wie veränderte Zeitressourcen (zeitlich), neue Tätigkeitsfelder (inhaltlich), Veränderungen in der Arbeitsteilung (sozial) oder emotionale Krisen (emotional), Dynamik im Medienrepertoire anstoßen und welche Faktoren für die Rezipierenden relevant sind, wenn sie ibre mediatisierte Lebensführung innerhalb der neuen Lebenssituation neu aushandeln.

Schlüsselwörter: Medienrezeption, Mediatisierung, Domestizierung, Medienethnographie, Medienrepertoire, Transitionen, Mediennutzung, Lebenskrise, Alltag

\section{The Mediatized Conduct of Everyday Life and its Change by Transitions}

\section{The Relevance of Transitional Periods for Audience and Reception Studies}

This article sets out to unfold the concept mediatized conduct of everyday life, an approach from which it is insightful to analyze change of media use in correlation to changes in the everyday lives of individuals such as separation, parenthood, or change of residence. As a distinctive feature, this concept divides everyday life into the following dimensions: temporal, spatial, social, content-related, emotional, meaningful, material, and physical. This allows for an accurate and systematic analysis of the connections between changes in the structure of everyday life to changes in media use. The empirical scope of the concept is demonstrated by the results of a qualitative panel-survey. The results reveal the ways in which specific everyday life driving forces such as shifts in the control over time (temporal), new interests and tasks (content-related), altered division of work in the relationship (social) or emotional crises (emotional), initiate dynamics in the media repertoire. Furthermore, it is shown which factors are relevant for the recipients if they negotiate their mediatized conduct of life within their new life situation.

Keywords: media reception, mediatization, domestication, media ethnography, media repertoire, transition, media use, life crisis, everyday life

* Dr. Stephan Niemand, Westfälische Wilhelms-Universität Münster, Institut für Kommunikationswissenschaft, Bispinghof 9-14, 48143 Münster, Deutschland, stephan.niemand@uni-muens ter.de, ORCID: https://orcid.org/0000-0001-9865-1757. 


\section{Einleitung}

Vor dem Hintergrund gesellschaftlicher Prozesse wie Enttraditionalisierung, Individualisierung und Pluralisierung der Lebensformen (Beck, 1986, 1996; Giddens,1996; Sennett, 1998) gewinnen Alltagsumbrüche als Analysekategorie an Bedeutung. Denn die Lebensverläufe der Menschen folgen nicht (mehr) einer universalistischen und linearen Abfolge des Lebens, wie sie der sogenannte Normallebenslauf (Kohli, 1985) noch postulierte, vielmehr ist die Biografie zunehmend von Brüchen und Diskontinuitäten geprägt (ausführlich dazu Niemand, 2020, S. 53-58). Wissenschaftliche Fachgebiete wie die Soziologie oder die Entwicklungspsychologie haben die Bedeutung von Alltagsumbrüchen im Sinne von Lebenskrisen, Übergangsphasen oder Wendepunkten für ihre Disziplin weitreichend analysiert. Im Kontrast dazu scheint die kommunikationswissenschaftliche Rezeptionsforschung ${ }^{1}$ bislang das enorme Potenzial zu übersehen, das Alltagsumbrüche wie Trennungen, Elternschaft oder Wohnungswechsel zur Analyse und Explikation der Medienrezeption und ihrem Wandel im Lebensverlauf bereitstellen.

Gegenwärtig gibt es nur sehr wenige kommunikationswissenschaftliche Studien, die Alltagsumbrüche und ihre Folgen für das Medienhandeln erforscht haben. Dies ist insofern überraschend, da weitgehend Einigkeit darüber besteht, dass die Nutzung von Medien immer in konkrete Alltagssituationen eingebettet ist (Röser, 2007) und somit die These naheliegt, dass eine tiefgreifende Veränderung der Alltagsstruktur auch einen Wandel des Medienhandelns anstößt. Wenn zum Beispiel nach der Geburt eines Kindes keine Zeit mehr für den Kinobesuch bleibt, wenn zu Beginn einer neuen Beziehung die Mediengewohnheiten des Partners oder der Partnerin übernommen werden oder wenn nach dem Renteneintritt neuerdings viel Fernsehen geschaut wird, dann ist die Dynamik im Medienrepertoire stets auf tiefgreifende Veränderungen in der Alltagsstruktur zurückzuführen. Besonders relevant erscheint dieses Themenfeld in Zeiten einer „deep mediatization" (Couldry \& Hepp, 2017, S. 53-56), weil die Verwobenheit zwischen Alltagshandeln und Medienhandeln gegenwärtig ein außerordentlich hohes Niveau aufweist. Als Ausgangspunkt für den Wandel der Mediennutzung wird hier also die Veränderung der Lebenssituation fokussiert und nicht - wie in der Kommunikationswissenschaft weit verbreitet - die Aneignung einer neuen Medientechnologie.

Der vorliegende Beitrag fragt danach, wie und warum Alltagsumbrüche Dynamik im Medienrepertoire anstoßen. Dazu wird in Abschnitt 2 zunächst der Forschungsstand aufgearbeitet. Hierbei wird deutlich, dass bislang ein theoretisches Konzept fehlt, um Veränderungen in der Alltagsstruktur systematisch mit einem Wandel der Mediennutzung erfassen und erklären zu können. Insofern liegt ein Schwerpunkt in dem Beitrag darauf, diese Lücke der kommunikationswissenschaftlichen Rezeptionsforschung zu schließen. Bezugnehmend auf verschiedene Theorien, die sich weitestgehend mit dem Konnex Alltag - Medien - Umbrüche befassen (Abschnitt 3), wird in Abschnitt 4 das

1 In diesem Artikel wird der Begriff Rezeptionsforschung weit gefasst. In Anlehnung an Röser (2016) wird Medienrezeption als Oberbegriff für drei Teilbereiche konzipiert: Mediennutzung, Medienaneignung und Medienwirkung. Der Schwerpunkt in diesem Artikel liegt im Bereich der Medienaneignung und hier speziell im Feld des situativen Medienhandelns (und damit nicht im Feld der Deutung von Medientexten durch Rezipierende). Mit dem Begriff Medienhandeln, der im weiteren Text häufig Verwendung findet, soll explizit betont werden, dass medienbezogenes kommunikatives Handeln stets in Alltagskontexten verankert ist und in Zusammenhang mit der sozialen Positionierung eines Subjekts in der Gesellschaft steht (siehe dazu auch Röser, 2007, S. 16). Die Relevanz von Alltagsumbrüchen für Aspekte der Mediennutzung und Medienwirkung wird durch diese Fokussierung aber keineswegs ausgeschlossen. 
Konzept der mediatisierten Lebensfübrung entfaltet. Mit entwickelt wurde dieses Konzept im Rahmen einer qualitativen Panelstudie zum Wandel des häuslichen Medienhandelns durch Alltagsumbrüche (Niemand, 2020). Anhand ausgewählter Befunde dieser Studie wird die empirische Reichweite des Konzepts der mediatisierten Lebensführung in Abschnitt 5 veranschaulicht. Die Einsichten geben Aufschluss darüber, welche Veränderungen in der Alltagsstruktur Dynamik im Medienrepertoire anstoßen und welche Faktoren für die Rezipierenden relevant sind, wenn sie ihre mediatisierte Lebensführung innerhalb der neuen Lebenssituation neu aushandeln.

\section{Forschungsstand: Dynamik im Medienhandeln im Zuge von Übergangsphasen}

Der Forschungsstand zum Wandel des Medienhandelns durch Alltagsumbrüche lässt sich als Forschungsdesiderat der kommunikationswissenschaftlichen Rezeptionsforschung charakterisieren. Dieser lässt sich in zwei Stränge systematisieren. Der erste Strang beinhaltet eine überschaubare Anzahl an Studien, die explizit Umbruchphasen wie den Renteneintritt und ihre Folgen für die Mediennutzung analysiert haben. Der zweite Strang umfasst hingegen allgemeiner alltagsbezogene und biografisch-orientierte Medienstudien, aus denen sich teils implizit Einsichten zum Themenfeld ableiten lassen. Grundsätzlich weist der Forschungsstand hinsichtlich der untersuchten Alltagsumbrüche sowie bezogen auf die analysierten Mediendienste eine hohe Varianz auf. Ferner nutzen die Autor*innen in ihren Studien unterschiedliche methodische Herangehensweisen (z. B. qualitative und quantitative Erhebungsverfahren) und beziehen sich auf verschiedene theoretische Konzepte (z. B. Uses-and-Gratification, Ansätze aus den Cultural Studies oder Bezüge zu Konzepten der Lebens- und Alltagswelt). Zentrale Analysen zum Forschungsstand und ihre wesentlichen Einsichten werden im Folgenden skizziert.

Die Befunde der wenigen Studien, die explizit zu Umbruchphasen und ibren Folgen für die Mediennutzung geforscht haben, belegen insgesamt einen starken Zusammenhang zwischen Alltagsstruktur und Medienhandeln. Gauntlett und Hill (1999, S. 79-109) zeigen beispielsweise - wenn auch wenig systematisch -, wie die Studienteilnehmer*innen nach verschiedenen Umbrüchen ihre Fernsehnutzungsmuster auf vielfältige Art und Weise verändern. Eine Rolle spielen dabei unter anderem soziale Aushandlungsprozesse mit dem Partner oder der Partnerin nach dem Beginn einer neuen Beziehung, Einsamkeit nach einem Umzug oder eine emotionale Krise nach einer Trennung. Mit Bezug zu digitalen Medien zeigt Augustin (2015), dass sich Studierende nach Aufnahme eines Auslandssemesters Weblogs neu aneignen und intensiv nutzen, um den Kontakt zu Familie und zum Freundeskreis auch über raum-zeitliche Distanzen halten zu können. Nach der Rückkehr verliert das Weblog hingegen seine Bedeutung, weil das soziale Umfeld nun wieder auf anderem Wege erreicht wird. Van Eimeren et al. (2017) belegen für den Berufseinstieg, dass sowohl der veränderte Tagesrhythmus als auch die körperliche Erschöpfung am Abend sowie der größere finanzielle Spielraum neue Mediennutzungsweisen anstoßen können. Ebenfalls für den Berufseinstieg stellt Karnowski (2003) mittels Sekundäranalyse des Datenmaterials des AGF/GfK-Fernsehpanels einen Rückgang der wöchentlichen Fernsehnutzungsdauer fest, obwohl die Fernsehnutzung am Wochenende anstieg. Für den Umbruch Elternschaft zeigen ihre Daten zudem, dass sich die Fernsehnutzungszeit nach der Geburt eines Kindes von 18-20 Uhr auf 20-23 Uhr und damit auf die Schlafenszeit der Kinder verschob. In einer Studie speziell zum Renteneintritt konstatieren Berger und Schönberger (2016), dass neben zusätzlichen Zeitressourcen auch der Wegfall berufsbezogener Anwendungen einen Einfluss auf die Veränderung der Mediennutzung haben kann. 
Alltagsbezogene und biografische Medienstudien interessieren sich zwar nicht in erster Linie für die Veränderung der Mediennutzung im Zuge von Alltagsumbrüchen, liefern durch ihre Fokussierung auf Medienhandeln in spezifischen Alltagskontexten und im biografischen Verlauf gleichwohl indirekt Einsichten zu diesem Zusammenhang. Neverla (1992) zeigt in ihrer älteren, gleichwohl beachtenswerten Studie, inwiefern das Zeiterleben im Alltag - oder anders ausgedrückt: die individuelle Temporalstruktur in einer spezifischen Lebenssituation - mit einer bestimmten Art und Weise der Fernsehnutzung korrespondiert. Während beispielsweise jüngere Menschen in Arbeitslosigkeit die Alltagszeit oft als leer und unausgefüllt empfanden und ihnen der Fernseher in dieser Lebensphase typischerweise als Beschäftigung diente, erlebten vor allem berufstätige Frauen mit Kindern Zeitknappheit im Alltag. Neben einer geringen Nutzung des Fernsehers griffen diese Frauen mehr als andere auf zeitökonomische Mediennutzungsstrategien zurück, um Zeit zu verdichten (z. B. durch Parallelnutzung von Medien) oder um Zeit zu dehnen (z. B. durch zeitversetztes Schauen mit Hilfe eines Videorekorders oder durch Vorspulen der Werbung). Neverlas Befunde verweisen folglich auf die Relevanz von Alltagsumbrüchen, weil sich im Zuge solcher Übergänge die wahrgenommene Verfügbarkeit von Zeit verändert, die die Nutzung von Medien maßgeblich prägt.

Auch Prommer (2009) verweist auf die hohe Bedeutung des Zeiterlebens im Alltag. So zeigt sie mittels medienbiografischer Interview-Daten, dass ein Großteil der Interviewten als zentralen Grund für den drastischen Rückgang ihrer Kinobesuche fehlende Zeit nach einer Familiengründung beziehungsweise einer beruflichen Veränderung angab. Ein anderer Aspekt zeigt sich in der medienbiografischen Studie von Hackl (2001). Durch den Auszug eines Kindes oder im Zuge einer Trennung wurde bei einigen Interviewten eine intensive eskapistische Fernsehnutzung ausgelöst, weil sie emotional mit der Situation überfordert waren. Hinsichtlich des Renteneintritts zeigen sich bei Hackl hingegen sehr unterschiedliche Befunde. So sind unter den Interviewten sowohl Personen, bei denen sich der Fernseher regelrecht zu einer Bezugsperson entwickelte, als auch Personen, bei denen die Fernsehnutzung auf niedrigem Niveau verblieb.

Insgesamt lassen sich aus dem Forschungsstand zwei zentrale Einsichten ableiten. Mit vergleichendem Blick auf die theoretische Rahmung offenbart sich erstens, dass die verwendeten theoretischen Konzepte nur eine eingeschränkte Erklärungskraft besitzen, um die dynamischen Prozesse, die sich im Medienhandeln über verschiedene Alltagsumbrüche hinweg zeigen, gegenstandsangemessen und umfänglich zu analysieren. So hebt zum Beispiel der mehrfach herangezogene Uses-and-Gratification-Ansatz (Berger \& Schönberger, 2016; Hackl, 2001; Karnowski, 2003; Prommer, 2009) lediglich darauf ab, Dynamik im Medienrepertoire über den Wandel der Bedürfnisstruktur zu erklären. Dabei gerät jedoch aus dem Blick, dass Dynamik jenseits von Bedürfnissen auch durch Einschränkungen wie fehlende Zeit für den Kinobesuch oder durch neue Handlungsoptionen wie ein gestiegenes Einkommen für den Kauf bestimmter Mediendienste entstehen kann. Mit dem Fehlen eines adäquaten theoretischen Konzepts geht zweitens einher, dass die Befunde keiner tiefergehenden Systematik folgen und es im Allgemeinen an empirischen Einsichten zum Zusammenhang zwischen Alltagsumbrüchen und einer veränderten Mediennutzung mangelt. Ein Zusammenhang, dies zeigen die wenigen Studien eindrücklich, besteht aber unverkennbar. Diese Prozesse zu ordnen und damit verschiedene Alltagsumbrüche systematisch zu charakterisieren, dazu soll der vorliegende Artikel einen wesentlichen Beitrag leisten. 


\section{Theoretischer Rahmen}

Zur Annäherung an den Zusammenhang zwischen Alltagsumbrüchen und dem Wandel des Medienhandelns liefert eine Kombination vier verschiedener Theorien vielversprechende Ergebnisse. Auf Basis der Ansätze Mediatisierung und Domestizierung (Abschnitt 3.1) wird zunächst das Verhältnis zwischen Alltag und Medien aus einer gesellschafts- und handlungstheoretischen Perspektive konzeptualisiert. Daran anschließend wird in Abschnitt 3.2 das soziologische Konzept der alltäglichen Lebensführung herangezogen, um die oftmals diffus verwendete Kategorie Alltag zu konkretisieren. Zudem wird hier das sozialpsychologische Transitionskonzept angeführt, um Merkmale und Verlauf von Alltagsumbrüchen näher zu bestimmen.

\subsection{Mediatisierung und Domestizierung}

Einen ersten Ausgangspunkt zur Analyse des Verhältnisses zwischen Alltag und Medien stellt der Mediatisierungsansatz dar. Dieser zielt auf die zunehmende Durchdringung verschiedener gesellschaftlicher Bereiche mit Medienkommunikation. Im Kontrast zu technikdeterministischen Ansätzen, die technologische Innovationen als wesentliche Ursache für einen Wandel der Gesellschaft ausmachen, wird im Mediatisierungsansatz davon ausgegangen, dass der Medienwandel in einer komplexen Wechselbeziehung mit gesellschaftlichen Entwicklungen steht und durch das medienbezogene Handeln der Menschen angestoßen wird (Krotz, 2001, 2007; Lundby, 2009). Mediendienste werden dabei nicht als eine abgeschlossene unveränderliche Technologie verstanden, sondern als etwas Prozesshaftes, über dessen Bedeutung auf verschiedenen Ebenen verhandelt und entschieden wird (Krotz, 2015). Konkret interessiert sich der Ansatz dafür, inwiefern sich im Zuge von Mediatisierungsprozessen das kommunikative Handeln der Menschen in verschiedenen Lebensbereichen des Alltags verändert (Krotz, 2012, S. 31-33). Wurden in der Vergangenheit zahlreiche Alltagstätigkeiten ohne Medienbezug ausgeführt, lassen sich im Zuge von Mediatisierungsprozessen gegenwärtig neue Formen und ein besonders hohes Niveau mediatisierter Alltagspraktiken ausmachen. Darauf verweisen gesellschaftliche Zeitdiagnosen einer „deep mediatization“ (Couldry \& Hepp, 2017, S. 53-56), einer „fortgeschrittene[n] Form von Dauervernetztheit des Individuums“ (Steinmaurer, 2013, S. 8) oder eines Subjekts, das sich stets in einem Zustand „permanently online, permanently connected“ befindet (Vorderer, 2015). Als Konsequenz der zunehmenden Verwobenheit zwischen medialem und nicht-medialem Handeln ist zeitgleich die Wahrscheinlichkeit gestiegen, dass sich Alltagsumbrüche vielschichtig auf die Nutzung von Medien auswirken.

Während in der Mediatisierungsforschung vor allem längere Zeiträume und große Entwicklungslinien von Wandel betrachtet werden, richtet sich die Domestizierungsforschung - im Sinne einer Präzisierung von Mediatisierung - auf konkretere Prozesse sich wandelnder Medienaneignung im Zuhause oder, weitgefasster, im Alltag (Röser et al., 2019, S. 27). Im Kern interessiert sich der Domestizierungsansatz für den Prozess, „wie Medien und Kommunikationstechnologien [...] im Aneignungsprozess Teil häuslicher Alltagsroutinen sowie Mittel sozialen Handelns werden“ (Röser \& Müller, 2017, S. 156). ${ }^{2}$ In der Analyse dieses Prozesses wird ersichtlich, wie der Wandel von Kommunikation durch neue Medientechnologien ermöglicht, aber erst von den Nutzer*innen

2 In der Domestizierungsforschung gibt es keine Übereinstimmung darüber, wo die Grenzen des Untersuchungsgegenstands verlaufen, ob beispielsweise ausschließlich die Mediennutzung im häuslichen Alltag von Interesse ist oder auch jenseits des Zuhauses. Ungeachtet dessen besteht 
alltagsbezogen und eigenwillig umgesetzt wird (Krotz, 2012; Röser et al., 2019). Die Veralltäglichung von Medien vollzieht sich über verschiedene Dimensionen: über die Anschaffung, die räumliche Platzierung bis hin zur Integration des Mediums in die alltäglichen Routinen und dem damit zusammenhängenden Wandel der kommunikativen Bezüge innerhalb verschiedener Lebensbereiche (Silverstone et al., 1992, S. 20-26; Röser \& Müller, 2017, S. 158). Dabei handelt es sich um einen diskontinuierlich verlaufenden Prozess, der zu keiner Zeit vollständig abgeschlossen ist. Zwar kann die Bedeutung von Medien in bestimmten Phasen eine gewisse Stabilität aufweisen, prinzipiell unterliegt diese aber stetig Veränderungsdynamiken. So können neben technologischen Innovationen wie der Entwicklung des Smartphones oder gesellschaftlichen Transformationen wie einer Novellierung medienrelevanter Gesetze speziell lebensweltliche Veränderungen wie Alltagsumbrüche Dynamik im Domestizierungsprozess auslösen (Röser et al. 2017, S. 145). Mit dem Begriff Re-Domestizierung beschreiben Röser et al. (2017, S. 146) eine hochdynamische Phase, in der „die Art und Weise der häuslichen Alltagsintegration eines oder mehrerer Medien neu verhandelt oder gestaltet wird.“

Der Domestizierungsansatz eröffnet folglich den Blick auf den (häuslichen) Alltag als bedeutungsvollen Kontext der Medienaneignung (Röser, 2007, S. 15). Problematisch ist allerdings, dass der Alltagsbegriff im Domestizierungsansatz „meist unscharf“ verwendet wird (Krotz \& Thomas, 2007, S. 31). Alltag wird zumeist gleichgesetzt mit dem Selbstverständlichen, dem Bekannten, dem Unhinterfragten oder dem Vertrauten und in diesem Zusammenhang auf die besondere Bedeutung von Habitualisierungen, Routinen oder Ritualen verwiesen (Silverstone, 1994, S. 18-19). Der Komplexität des Alltags wird ein solches Verständnis nicht gerecht. Dies ist mindestens aus drei Punkten problematisch: Erstens besitzt ein solches Begriffsverständnis kaum einen analytischen Mehrwert. Zweitens leidet unter der diffusen Verwendung des Alltagsbegriffs die Vergleichbarkeit verschiedener Medienaneignungsstudien. Schließlich erschwert es drittens, den Zusammenhang zwischen Alltagshandeln und Medienhandeln zu theoretisieren, weil unklar ist, was den Alltag eigentlich ausmacht und inwiefern er mit der Mediennutzung zusammenhängt. Diese Problematik der diffusen Begriffsverwendung wird im Folgenden aufgelöst.

\subsection{Alltägliche Lebensführung und Übergangsphasen}

Mit dem elaborierten soziologischen Konzept der alltäglichen Lebensführung (Voß, 1991) lässt sich die Kategorie Alltag präzisieren und gewinnbringend auf das Feld der kommunikationswissenschaftlichen Rezeptionsforschung übertragen. Alltägliche Lebensführung wird verstanden, als „der Ort, wo alles zusammenkommt“ (Jurczyk \& Rerrich, 1993). In dem Konzept wird der Anspruch verfolgt, das Leben in seiner gesamten Breite zu erfassen. Von Interesse sind sämtliche Tätigkeiten, die eine Person im Rahmen ihrer Lebensführung ausführt. Dabei wird in besonderer Weise fokussiert, wie das Handeln in unterschiedlichen Lebensbereichen in seiner Gesamtheit koordiniert wird:

„Ist eine Person beispielsweise erwerbstätig, hat Familie, pflegt regelmäßig Kontakt zu Freunden und Verwandten, ist Mitglied in einem Verein und zudem in einem Verband (etwa einer Gewerkschaft) tätig usw., dann bildet das, was wir Lebensführung nennen, einen strukturierenden und

Einigkeit darin, dass die Wechselbeziehung zwischen medialem und nicht-medialem Handeln analysiert werden muss, indem die Mediennutzung stets in Rückbezug zum alltäglichen Kontext erhoben wird (Niemand, 2020: 30-31). 
koordinierenden Rahmen für alle in diesen Feldern regelmäßig ausgeübten Handlungen.“ (Voß, 2001, S. 204)

Als besonders instruktiv erweist sich, dass das Gesamtkonstrukt Lebensführung im Sinne einer forschungsleitenden Heuristik in verschiedene Strukturdimensionen differenziert wird:

„Die Form der Lebensführung einer Person besteht darin, zu welchen Zeitpunkten, an welchen Orten, in welcher inhaltlichen Form, in welchen sozialen Zusammenhängen und orientiert an welchen sozialen Normen, mit welchen sinnhaften Deutungen sowie mit welchen Hilfsmitteln oder Ressourcen und schließlich mit welchen emotionalen Befindlichkeiten eine Person im Verlauf ihres Alltags typischerweise tätig ist." (Voß, 1995, S. 32, Herv. i. O.)

Die Lebensführung kann demnach in zeitlicher, räumlicher, inhaltlicher, sozialer, sinnbezogener, materieller, emotionaler - und auch körperlicher (Niemand, 2020, S. 43, 224) - Hinsicht präzise beschrieben werden. In dem in Abschnitt 4 entfalteten Konzept der mediatisierten Lebensführung werden diese Dimensionen auf das Feld der kommunikationswissenschaftlichen Rezeptionsforschung überführt, um aufbauend auf dieser Differenzierung eine fruchtbare Analyseperspektive auf den Gesamtzusammenhang von Alltagstätigkeiten und der darin eingelagerten Mediennutzung bereitzustellen.

Jenseits der Systematisierung der Dimensionen von Alltag stellt sich die Frage, was einen Alltagsumbruch kennzeichnet und welche zentralen Verlaufsmerkmale ein Umbruch aufweist. Hier liefert das sozialpsychologische Transitionskonzept von Welzer (1993a) wichtige Impulse. Transitionen sind Phasen im Leben eines Menschen, die sich durch eine hohe Intensität neuer Erfahrungen auszeichnen, also Übergänge „von Individuen aus eingelebten Lebensabschnitten und -zusammenhängen in andere“ (Welzer, 1993b, S. 137), wie der Wechsel des Wohnortes, ein Partnerverlust oder ein neuer Karriereabschnitt. Transitionen werden in dem Konzept weder als eine biografisch vorbestimmte lineare Abfolge von Ereignissen mit festen Ausgangs- und Endpunkten sowie mit einer jeweils spezifischen Bewältigungsanforderung verstanden, noch als rein individuenzentrierte Prozesse (Welzer, 1993a, S. 13-38). Vielmehr handelt es sich bei einer Transition um einen Prozess, der in einem in permanentem Wandel befindlichen Lebenslauf eine Vielzahl an Veränderungen in unterschiedlichen Lebensbereichen nach sich zieht und sich teils mit weiteren Übergängen überblendet und interagiert. Transitionsprozesse gelten folglich als nie vollständig abgeschlossen und können unterschiedliche Entwicklungsverläufe nehmen. Inwiefern Transitionen beziehungsweise Alltagsumbrüche auch das Medienhandeln prägen, wird folgend im Konzept der mediatisierten Lebensführung präzisiert.

\section{Zum Konzept der mediatisierten Lebensführung und ihrem Wandel durch Alltagsumbrüche}

Die Kombination der vier theoretischen Zugänge hat sich bei der Durchführung einer qualitativen Panelstudie zum Wandel der häuslichen Mediennutzung durch Alltagsumbrüche als instruktiv für die Analysen erwiesen (Niemand, 2020). Basierend auf den skizzierten Theoriesträngen sowie auf den empirischen Befunden der Panelstudie wird in diesem Abschnitt das Konzept der mediatisierten Lebensfübrung entfaltet. Dieses stellt eine Heuristik für empirische Analysen zur Veränderung des Medienhandelns im Zuge von Alltagsumbrüchen bereit und schließt folglich das oben identifizierte Theoriedefizit. Die Kernideen des Konzepts lassen sich entlang von fünf Prämissen skizzieren (zur Herleitung auch Niemand, 2020, S. 233-252). 
1. Die mediatisierte Lebensfübrung erfolgt sinnhaft in Rückbezug zu den für eine

Person relevanten Lebensbereichen und umfasst das gesamte Medienrepertoire.

Der Begriff mediatisierte Lebensführung bringt sprachlich zum Ausdruck, dass Medien(technologien) ihre Bedeutung erst durch das sinnhafte Handeln innerhalb der für ein Subjekt relevanten Lebensbereiche entfalten. Medien werden beispielsweise genutzt, um berufliche Anforderungen zu erfüllen (Erwerbsarbeit), eine angenehme Zeit mit der Partnerin oder dem Partner zu verbringen (privater Nahbereich) oder um persönliche Interessen zu verfolgen (Freizeit und Hobbies). Wieso eine Person sich bestimmten Medien zuwendet, andere ablehnt, welche Inhalte sie auswählt und wie diese verarbeitet und gedeutet werden, wird erst durch den Rückbezug zur spezifischen Lebenssituation des jeweiligen Menschen erklär- und verstehbar. Insofern ist die spezifische Lebenssituation der entscheidende Handlungsrahmen, in dem Medien ihre Bedeutung entfalten. Ist die mediatisierte Lebensführung erst einmal erschaffen und eingerichtet, weist sie eine relativ stabile Struktur auf und gewährleistet somit Handlungssicherheit und Handlungsentlastung, weil ein Subjekt in wiederkehrenden Situationen nicht ständig neu entscheiden muss, wie es Medien sinnvoll und angemessen nutzen kann oder soll.

Im Einklang mit dem Anspruch, die Breite des Lebens einzubeziehen, umfasst die mediatisierte Lebensführung sämtliche Alltagstätigkeiten, die mit Medien(technologien) ausgeführt werden und folglich das gesamte Medienrepertoire (Hasebrink \& Domeyer, 2012). Medien werden dabei im Sinne einer „double articulation“ (Silverstone \& Haddon, 1996, S. 62) einerseits als Träger von symbolischen Bedeutungen sowie andererseits als technologische Artefakte konzipiert, d. h. es ist sowohl von Interesse, welche Inhalte rezipiert, als auch, wie Medien als materielle Objekte in die Routinen und Gewohnheiten des Alltags integriert werden. Dabei handelt es sich gleichwohl nur um eine analytische Trennung, denn ohne die jeweils andere Ebene kann die Aneignung eines Mediums nicht gedacht werden (Hartmann, 2013, S. 25-26).

\section{Die mediatisierte Lebensfübrung ist analytisch in verschiedene Strukturdimensio-} nen aufgeschlüsselt.

Eine zentrale Besonderheit des Konzepts ist, dass die Lebensführung im Sinne einer forschungsleitenden Heuristik analytisch in verschiedene Strukturdimensionen (Voß, 1995, S. 32) aufgeschlüsselt ist. Als relevant haben sich die folgenden Dimensionen erwiesen:

\section{Tabelle 1: Dimensionen der mediatisierten Lebensfübrung}

\begin{tabular}{ll}
\hline zeitlich: & zum Beispiel Zeitsouveränitäten und Tagesrhythmen \\
räumlich: & zum Beispiel Raumarrangements und Bewegungsmuster \\
sozial: & $\begin{array}{l}\text { zum Beispiel (partnerschaftliche) Abstimmungsprozesse und } \\
\text { (geschlechtsgebundene) Arbeitsteilungen }\end{array}$ \\
inhaltlich: & zum Beispiel Interessensgebiete und Tätigkeitsfelder \\
sinnbezogen: & zum Beispiel Lebensentwürfe, Orientierungsmuster und Leitbilder \\
emotional: & zum Beispiel emotionale Krisen \\
materiell: & zum Beispiel technologische Ausstattung und finanzielle Ressourcen \\
körperlich: & zum Beispiel Gesundheit und Krankheit \\
\hline
\end{tabular}

Quelle: Eigene Darstellung 
Die jeweiligen Ausgestaltungen innerhalb der Dimensionen stehen in Zusammenhang mit spezifischen Medienpraktiken, sie sind der Kontext der Mediennutzung. Zwar determinieren die Ausprägungen der Lebensführung das Medienhandeln nicht, sie stellen aber im Sinne einer Verwobenheit zwischen Medien- und Alltagshandeln bedeutsame Rahmenbedingungen für die Medienaneignung bereit. So macht es zum Beispiel für die Nutzung von Medien einen Unterschied, ob zu Kernarbeitszeiten oder im Schichtdienst gearbeitet wird (zeitlich), ob man eine Fernbeziehung führt oder zusammenwohnt (räumlich), ob man sich in einer emotionalen Krise befindet (emotional) oder ob die Hörfähigkeit eingeschränkt ist (körperlich).

3. Alltagsumbrüche sind non-lineare, verlaufsoffene und reflexiv gestaltete Übergangsphasen. Sie stoßen Wandel im Medienrepertoire an, der Momente von Dynamik und Beharrung umfasst.

Zur Explikation, inwiefern Medien- und Alltagshandeln miteinander verwoben sind und warum sich die Mediennutzung im Laufe eines Lebens ändert, erweisen sich Alltagsumbrüche als zielführende Analysekategorie. Alltagsumbrüche lassen sich definieren als non-lineare, verlaufsoffene und reflexiv gestaltete Übergangsphasen, die sich durch eine hohe Intensität neuer Erfahrungen auszeichnen und einen Wandel der mediatisierten Lebensführung anstoßen können. ${ }^{3}$ Non-linear und verlaufsoffen sind Übergangsphasen, weil vermeintlich identische Umbrüche unterschiedlich erlebt werden können (Welzer, 1993a, S. 294-295). Wie die mediatisierte Lebensführung vor dem Hintergrund der veränderten Alltagsstruktur angepasst wird, ist keineswegs vordefiniert, sondern unterliegt vielmehr der subjektiven Wahrnehmung und der eigensinnigen Adaption der Mediennutzer\%innen. Denn im Gegensatz zu einem Determinationsverständnis wird die mediatisierte Lebensführung „als relativ autonome ,Reaktion“ von Personen“ (Voß, 1995, S. 37) verstanden, die aktiv konstruiert wird. Folglich gibt es nicht den Alltagsumbruch mit dem Effekt auf das Medienhandeln, sondern es können sich nach einem ähnlichen Alltagsumbruch - je nachdem, welche Ziele gesetzt werden und wie die neue Lebenssituation bewertet wird - unterschiedliche Entwicklungsverläufe im Medienrepertoire zeigen. Grundsätzlich wird in der Zeit des Übergangs besonders aufmerksam geprüft, welche Handlungsoptionen ein Mensch wahrnehmen will und welche Medien für die Alltagsgestaltung als sinnvoll empfunden werden. Alltagsumbrüche zeichnen sich daher durch eine auffallend bohe Reflexivität aus.

Auch wenn hier also die Devise von Gauntlett und Hill (1999, S. 283) gilt: „everyone is a bit different, everything is very complex", so sind diese Prozesse gleichwohl nicht willkürlich. Vielmehr kann davon ausgegangen werden, dass sich ähnliche Muster zeigen, wie Menschen den Übergang von einer Lebenssituation in eine andere mit Medien gestalten. Der Wandel der mediatisierten Lebensführung lässt sich dabei als Zusammenspiel von Dynamik und Beharrung konzipieren (Röser et al., 2017). Während der Begriff Dynamik auf die „fortlaufende, zeitweise auch schubhafte Veränderung im [...] Medienhandeln“ abhebt, wird kontrastierend dazu mit dem Begriff der Beharrung „das Festhalten an bewährten Medienpraktiken, zum Beispiel durch das Im-Spiel-Halten der alten (analogen) Medien“ gefasst (Röser et al., 2017, S. 140-141). Umbrüche lösen einerseits schubhafte Neuerungen des Medienhandelns und damit dynamische Prozesse aus, andererseits verlieren in diesem Prozess nicht sämtliche Medienpraktiken im Medienre-

3 Analytisch lassen sich Alltagsumbrüche in vorhersehbare (z. B. Heirat oder Geburt eines Kindes) und unvorhersehbare Ereignisse (z. B. Autounfall oder Lottogewinn) differenzieren (Welzer, 1990, S. 43). Während die Bewältigungsanforderungen bei vorhersehbaren Alltagsumbrüchen vorbereitet werden können, ist dies bei unvorhersehbaren (eher) nicht der Fall. 
pertoire an Bedeutung. Vielmehr beharren die Nutzer*innen auf bewährten Medienpraktiken, solange diese für ihre Alltagsgestaltung als sinnvoll empfunden werden (Röser et al., 2019, S. 30-32).

Entlang der Strukturdimensionen wird - und dies ist die zentrale Stärke des Konzepts - systematisch und präzise sichtbar, inwiefern spezifische Alltagsveränderungen Dynamik im Medienrepertoire auslösen und wieso bestimmte Mediennutzungspraktiken trotz des Umbruchs ihre Sinnhaftigkeit behalten. Auf diese Weise wird evident, was sonst eher verborgen und unbewusst verbleibt. In Form von alltagsspezifischen Antriebsfaktoren liefern die Veränderungen innerhalb der Alltagsstruktur Aufschluss über Hintergründe und Erklärungszusammenhänge für den Wandel der mediatisierten Lebensführung. Inwiefern steht die Dynamik im Medienrepertoire zum Beispiel in Zusammenhang mit veränderten Zeitsouveränitäten (zeitlich)? Welche Rolle spielen neue Tätigkeitsfelder (inhaltlich) oder ein Wandel der Orientierungsmuster (sinnbezogen)? Und inwiefern macht sich eine emotionale Belastung (emotional) oder eine einschneidende Veränderung des Gesundheitszustands (körperlich) bemerkbar? All dies sind Fragen, die sich durch die empirische Analyse von Alltagsumbrüchen und ihren Folgen für das Medienhandeln präzise entlang der Strukturdimensionen beantworten lassen. Auch bei sich zeitlich überblendenden Übergangsphasen - wenn beispielsweise der Berufsbeginn mit einem Umzug und der Trennung von der Freundin oder dem Freund einhergeht - lässt sich systematisch herausarbeiten, inwiefern sich die Alltagsstruktur konkret verändert und warum in diesem Zusammenhang die mediatisierte Lebensführung angepasst wird.

\section{Die (Neu)Konstitution der mediatisierten Lebensfübrung erfolgt im Spannungsfeld zwischen Entfaltungschancen, Zwängen und Erwartungen sowie Bedürfnissen und Daseinsthemen.}

Die jeweiligen Alltagsveränderungen im Zuge eines Übergangs finden ihren Ausdruck in einem Wandel der Entfaltungschancen, Zwängen und Erwartungen sowie Bedürfnissen und Daseinsthemen. Insofern entscheidet über Dynamik und Beharrung innerhalb der mediatisierten Lebensführung, wie mit diesen Faktoren umgegangen wird und wie diese im Medienhandeln wirksam werden.

Der Begriff Entfaltungschancen hebt dabei auf das „Ressourcenpotenzial“ (Voß, 1991, S. 263) ab, das spezifische Lebensbereiche zur Gestaltung der mediatisierten Lebensführung bereitstellen. Um ein einfaches Beispiel zu nennen: Der Einstieg in einen Beruf mit Gleitzeit oder mit der Option, im Home Office arbeiten zu können, bietet andere Möglichkeiten, die Tagesstruktur zu gestalten, als Berufe, in denen dies nicht möglich ist und die somit weniger Entfaltungschancen gewähren. Die Begriffe Erwartungshaltung und Zwänge beschreiben die „lebenspraktische Begrenzung der existenziell möglichen Vielfalt" (Voß, 1991, S. 260). Dabei kann es sich zum Beispiel um Rollenanforderungen oder um gesellschaftliche Leitbilder handeln, die mehr oder weniger abstrakt nahelegen, wie man sich als Eltern, Berufstätiger, Nachbarin, Partner, Frau, Mann usw. in spezifischen Situationen (nicht) verhalten soll, und die somit im (Medien)Handeln wirksam werden können.

Der Begriff der Bedürfnisse lässt sich prinzipiell als Zustand des Mangels verstehen, nach dessen Ausgleich, bewusst oder auch unbewusst, gestrebt wird (Bergius, 2009, S. 114). Bedürfnisse können prägend für die Mediennutzung sein, insofern finden mit dem Begriff der Bedürfnisse die Überlegungen des Uses-and-Gratification-Ansatzes in dem Konzept Berücksichtigung, ohne diese aber als alleinigen Erklärungsfaktor für die Konstruktion der mediatisierten Lebensführung anzusehen. Daseinsthemen lassen sich als „Basisthemen“ verstehen, die in einer bestimmten Lebenssituation relevant werden 
und die als (geäußerte oder beobachtbare) „Gedanken, Wünsche, Befürchtungen, Hoffnungen und Konflikte“ (Thomae, 1988, S. 53) sichtbar werden. Im Sinne der strukturanalytischen Rezeptionsforschung handelt es sich dabei um handlungsleitende Themen, wie besondere persönliche Anliegen (z. B. Hobbys oder situative Interessen) oder allgemeine gesellschaftlich relevante Themen (z. B. Entwicklungsaufgaben), die die Medienrezeption maßgeblich beeinflussen (Charlton und Neumann, 1992; Neumann-Braun, 2005).

Bei der Neustrukturierung geht es für ein Subjekt gerade darum, wie sich - aus seiner Perspektive - die veränderten Entfaltungschancen, Zwänge und Erwartungen sowie Bedürfnisse und Daseinsthemen zur gesamten mediatisierten Lebensführung verhalten und wie es diese zu einem zufriedenstellenden Gesamtkonstrukt verbindet. Als besondere Herausforderung erweist sich dabei, dass die verschiedenen Lebensbereiche in einem komplexen Wechselverhältnis zueinander stehen. Die mediatisierte Lebensführung muss so arrangiert werden, dass die Anforderungen aus dem Beruf, der Partnerschaft, dem Freundes- und Bekanntenkreis usw. möglichst zufriedenstellend miteinander in Einklang gebracht werden. Eine Rolle spielt zudem, dass ein Übergang zumeist von der Kompensation von Verlusten und der Wiedergewinnung von bereits Erreichtem geprägt ist (Welzer, 1993a, S. 287-288). Damit ist keineswegs gemeint, dass der „alte“ Zustand wiederhergestellt werden muss, aber das Handeln ist immer auch geprägt von der Differenz der neuen Lebenssituation im Kontrast zur vergangenen.

\section{Die mediatisierte Lebensführung ist zwischen Struktur und Handlung angesiedelt und stellt einen Mikrokosmos des gesellschaftlichen und kulturellen Wandels durch Medien dar.}

Die mediatisierte Lebensführung wird als „Mikrokosmos des gesellschaftlichen und kulturellen Wandels durch Medien“ verstanden, in dem „alle gesellschaftlichen und kulturellen Fragen der Medienkommunikation ihren Ausdruck [finden]“ (Röser, 2007, S. 21). Die Mikroebene wird somit nicht als Gegensatz zur Makroebene konzipiert, vielmehr lässt sich mit Blick auf den Wandel der mediatisierten Lebensführung analysieren, inwiefern Medienpraktiken mit gesellschaftlichen Rahmenbedingungen interagieren (zum Beispiel bezogen auf die Kategorie Geschlecht: Röser, 2007; Roth \& Röser, 2019).

Unterschieden werden zwei Ebenen gesellschaftlicher Rahmenbedingungen: Erstens die Medienumgebung, die als Gesamtarrangement von Medien durch Mediatisierungsprozesse einem permanenten Wandel unterliegt (Livingstone, 2015; Meyrowitz, 1995). Die gegenwärtige Medienumgebung prägt die auf der Mikroebene aktiv konstruierte mediatisierte Lebensführung. Denn je nach historischem Zeitpunkt stehen den Menschen unterschiedliche Mediendienste zur Verfügung, die sie für ihre Alltagsgestaltung heranziehen können. Die Veränderungen, die Alltagsumbrüche im Medienhandeln anstoßen, variieren daher auch in Verbindung mit dem Mediatisierungsgrad, weil je nach Zeitpunkt bestimmte Alltagshandlungen mediatisiert ausgeführt werden oder eben (noch) nicht. Zweitens wird die Konstruktion der mediatisierten Lebensführung von gesellschaftlichen Verhältnissen, Leitbildern und Diskursen wie gesetzlichen Rahmenbedingungen, Geschlechterordnungen oder milieuspezifischen Ungleichheiten geprägt. Daraus folgt: Neben einem Wandel der Alltagsstruktur durch Umbrüche - wie er hier im Vordergrund steht - kann Dynamik in der mediatisierten Lebensführung auch durch technologische Innovationen (wie zum Beispiel die Verbreitung und Durchsetzung des Smartphones) oder durch gesellschaftliche Veränderungen (wie zum Beispiel der Lockdown während der Corona-Pandemie) angestoßen werden.

Grundsätzlich gilt: Die Konstruktion der mediatisierten Lebensführung ist nicht einfach eine Folge struktureller Lebensbedingungen, sondern sie wird durch die Person 
unter aktiver Verarbeitung der Lebensumstände geschaffen (Voß, 1991, S. 265, 306). Die Medienumgebung sowie die gesellschaftlichen Verhältnisse, Leitbilder und Diskurse sind demnach Bedingungen des individuellen Handelns, determinieren es aber nicht. In diesem Sinne handelt es sich bei der mediatisierten Lebensführung um ein sozialkonstruktivistisches Konzept, das in einer vermittelnden Position zwischen Struktur (sozialobjektivistische Konzepte) und Handlung (subjektiv-interpretative Ansätze) verortet ist. Entsprechend lassen sich anhand der konkreten Medienpraktiken immer auch Aussagen zu gesellschaftlichen Machtkonstellationen oder sozialen Ungleichheiten treffen.

Modellhaft lässt sich der Wandel der mediatisierten Lebensführung durch Alltagsumbrüche wie in Abbildung 1 darstellen.

\section{Empirische Reichweite des theoretischen Konzepts}

Entlang der Befunde der oben bereits erwähnten ethnografisch-orientierten Panelstudie zum Wandel des häuslichen Medienhandelns durch Alltagsumbrüche (Niemand, 2020), wird in diesem Abschnitt der analytische Mehrwehrt des Konzepts der mediatisierten Lebensführung veranschaulicht. Die Analyse von Alltagsumbrüchen war ein wichtiger Teilaspekt der größer angelegten DFG-geförderten Projektreihe „Das mediatisierte Zuhause“ (Röser et al., 2019), in der die Domestizierung des Internets und der dazugehörige Wandel des Medienrepertoires sowie der häuslichen Kommunikationskulturen über einen Zeitraum von acht Jahren in 25 Paarhaushalten vielschichtig untersucht wurde. ${ }^{4}$ Kennzeichnend für die Projektreihe waren drei zentrale Besonderheiten: Erstens erfolgte die Analyse der Medienaneignung im Kontext des häuslichen Alltags der Paare, um das Zusammenspiel zwischen medialem und nicht-medialem Handeln herauszuarbeiten. Zweitens wurde das gesamte Medienrepertoire berücksichtigt, so dass Aussagen zu Veränderungen der Internet-, Fernseh-, Radio- und Zeitungsnutzung getroffen werden konnten. Und schließlich war drittens das Längsschnittdesign der Studie prägend, da es erlaubte, Wandel prozessorientiert im Zeitverlauf empirisch zu erfassen. Im Folgenden werden die methodische Herangehensweise sowie die Befunde der Studie zum Teilaspekt Alltagsumbrüche erläutert.

\subsection{Datenmaterial: Qualitative Panelstudie zum Wandel des häuslichen Medienhandelns durch Alltagsumbrüche}

Im Zentrum der Studie stand die Fragestellung, inwiefern Alltagsumbrüche das häusliche Medienhandeln dynamisieren und welche Aussagen sich anhand dieser Analysen zur Beziehung zwischen Lebensführung und Medienhandeln treffen lassen (Niemand, 2020, S. 3). ${ }^{5}$ Dazu wurde zu vier Erhebungszeitpunkten (2008, 2011, 2013 und 2016) untersucht, in welchen der 25 Paarhaushalten der gesamten Projektreihe sich welche Alltagsumbrüche ereigneten und wie sich in diesem Zusammenhang das häusliche Medienhandeln veränderte. Konkret interessierte uns der Wandel der Radio-, Fernseh-, Print- und

4 Das Sample der Projektreihe war systematisch in drei Alters- und zwei Schulbildungsgruppen quotiert und umfasste Menschen aus verschiedenen Lebensphasen, Berufsgruppen und Lebenslagen (siehe zur Übersicht über das Sample Röser et al., 2019, S. 44-48). Jenseits der Erforschung von Alltagsumbrüchen umfasste die gesamte Projektreihe fünf weitere zentrale Analysefelder (siehe zur Differenzierung des Erkenntnisinteresses Röser et al., 2019, S. 42-44).

5 Der Fokus lag folglich auf Dynamik im Medienrepertoire. Gleichwohl ist die Frage nach Beharrungsmomenten eine relevante Perspektive, insbesondere, weil das Festhalten an etablierten Mediennutzungsmustern in Umbruchphasen Stabilität und Rückhalt geben kann. 


\section{Abbildung 1: Analysemodell: Wandel der mediatisierten Lebensfübrung durch Alltagsumbrüche}

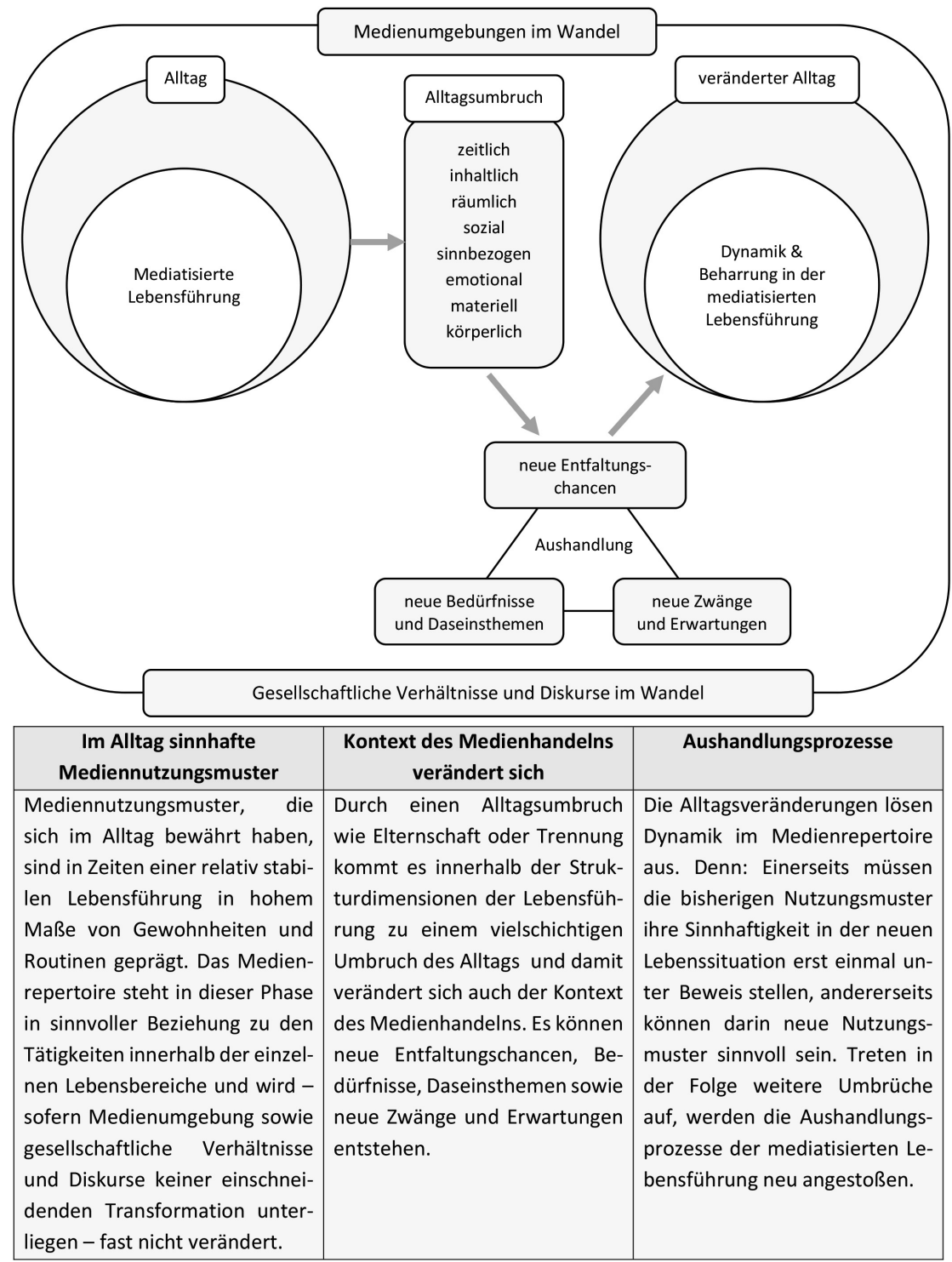

Quelle: Erweiterte Darstellung in Anlehnung an Niemand, 2020, S. 70, 239

Onlinenutzung. Im Sinne einer medienethnografisch-orientieren Herangehensweise kam dabei ein Methodenmix zum Einsatz, um möglichst ein ganzheitliches Verständnis über den Forschungsgegenstand zu entwickeln (Bachmann \& Wittel, 2006). Das Herzstück bildeten Leitfadeninterviews im Zuhause der Paare, die durch schriftliche Befra- 
gungen, Fotodokumentationen, Kartensortierungstechniken und weitere Methodenelemente ergänzt wurden. Je nachdem, welche Alltagsumbrüche sich in dem Haushalt ereigneten, wurden die Erhebungsinstrumente gezielt angepasst. Welche Ereignisse als Umbrüche identifiziert wurden, überließen wir überwiegend den Einschätzungen der Paare, indem wir sie in den Interviews und in den schriftlichen Befragungen nach einschneidenden Alltagsveränderungen fragten. Dies ist sinnvoller als eine deduktive Vorgehensweise, bei der im Vorfeld festgelegt wird, welche Alltagsumbrüche untersucht werden, weil die Interviewten „in der Regel deutlicher bestimmen können, ob ihr Alltag im Prinzip stabil ist, sich in einer Umbruchsituation befindet oder eine neue Struktur bekommen hat" (Voß, 1991, S. 356).

Ausgewertet wurden die Daten mittels ethnografisch-orientierter Porträts, einem Verfahren, das eine besonders kontextsensitive Analyse erlaubt und „regelgeleitet, transparent und intersubjektiv nachvollziebbar abläuft" (Röser et al., 2018, S. 201, Herv. i. O.). Dazu wurden in einem ersten Schritt die Einzelfälle in Form von Porträts systematisch verdichtet. Ein besonderes Augenmerk lag darauf, für jeden Umbruch wie Elternschaft oder Trennung eine separate Porträtstruktur zu entwickeln, weil die jeweiligen Umbrüche spezifische Veränderungen der Alltagsstruktur mit sich bringen, die es mit dem Wandel des Medienhandelns in Beziehung zu setzen galt. In einem zweiten Schritt wurden alle Fälle des „gleichen“ Alltagsumbruchs einer vergleichenden Analyse unterzogen, um Muster und Entwicklungsverläufe im Erleben des Umbruchs und den daraus folgenden Dynamiken im Medienhandeln herauszuarbeiten (transitionsspezifischer Fallvergleich). Schließlich erfolgte in einem dritten Schritt ein transitionsübergreifender Fallvergleich, indem jenseits des einzelnen Alltagsumbruchs entlang der Dimensionen der mediatisierten Lebensführung untersucht wurde, inwiefern alltagsspezifische Prozesse wie Umstrukturierungen des Tagesrhythmus (zeitlich) oder Veränderungen in der Arbeitsteilung (sozial) Dynamik im Medienrepertoire angestoßen haben (siehe ausführlicher zur methodischen Herangehensweise Niemand, 2020, S. 89-110).

Das Sample umfasste 22 Paarhaushalte, in denen sich im Erhebungszeitraum Alltagsumbrüche ereigneten. Da sich drei Paare getrennt hatten und seitdem unabhängig voneinander einen eigenen Haushalt führten, wurden letztlich 25 Haushalte untersucht. Tabelle 2 gibt einen Überblick über die Verteilung der Alltagsumbrüche.

Das Ausmaß der Alltagsveränderung durch einen Umbruch wurde aus Sicht der Paare unterschiedlich intensiv erlebt (Niemand, 2020, S. 113-116). Den Wandel durch Elternschaft, Trennung und Beginn einer neuen Partnerschaft sowie Umzug, Auszug eines Kindes oder Tod des Partners oder der Partnerin erlebten unsere Interviewten weitgehend als tiefgreifende Veränderung der Alltagsstruktur. Hier kam es in mehreren Dimensionen der mediatisierten Lebensführung zu wesentlichen Veränderungen, und hier wurden vielschichtige und weitreichende Zusammenhänge zwischen Umbruch und Dynamik im Medienrepertoire sichtbar. Die Alltagsumbrüche Renteneintritt, berufliche Veränderungen sowie gesundheitliche Beeinträchtigungen wurden von den Interviewten in unserem Sample hingegen eher nicht als tiefgreifende Einschnitte empfunden, weil zum Beispiel vor dem Renteneintritt „nur“ eine geringfügige Beschäftigung ausgeübt wurde, oder weil nach dem Wechsel des Arbeitgebers die berufliche Kerntätigkeit erhalten blieb und es folglich in den Dimensionen der mediatisierten Lebensführung zu wenigen und nicht so weitreichenden Veränderungen kam. Zweifellos sind auch diese Formen eines Umbruchs als tiefgreifende Einschnitte möglich, wenn beispielsweise der Renteneintritt aus einer Vollzeittätigkeit erfolgt oder wenn die Gesundheit beispielsweise durch den Verlust der Hörfähigkeit schwerwiegender beeinträchtigt wird. Es erscheint daher lohnenswert, die in unserem Sample weniger einschneidenden Umbrüche 
in weiteren Analysen auch als tiefergehende Einschnitte zu untersuchen. Gleichwohl führten auch diese weniger tiefgreifenden Umbrüche zu Dynamik.

\section{Tabelle 2: Alltagsumbrüche im Sample zwischen 2008 und 2013: Übersicht}

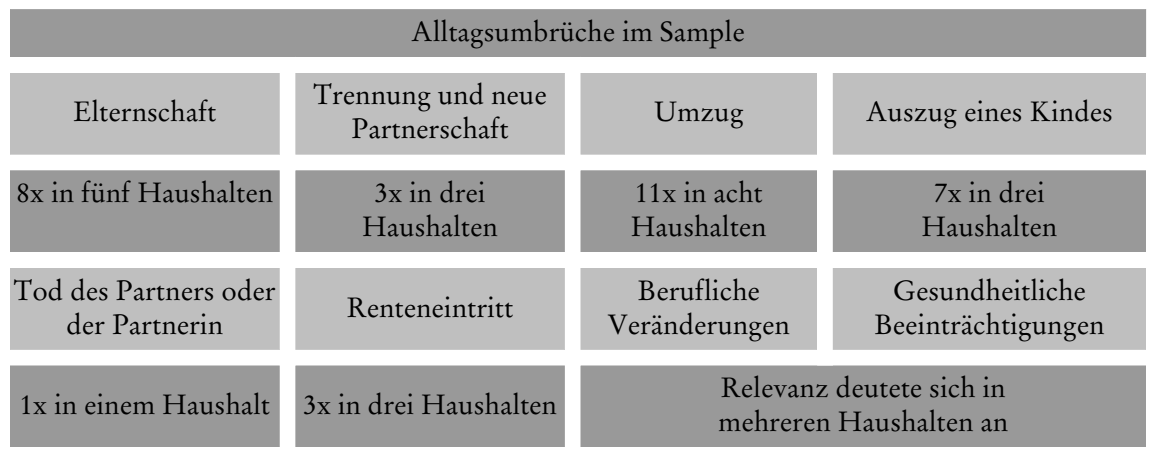

Quelle: Niemand, 2020, S. 114, gekürzt

\subsection{Veränderungen in den Alltagsdimensionen als Antriebsfaktor für Dynamik im Medienrepertoire}

In der Studie wurde für jeden Alltagsumbruch dezidiert analysiert, inwiefern sich durch den Wandel der Alltagsstruktur das Medienhandeln veränderte. Grundsätzlich folgt die angestoßene Dynamik im Medienrepertoire keinem Determinismus, weil die Alltagsveränderungen teils leicht unterschiedlich erlebt werden und weil die Paare ihr Medienhandeln in gewissem Maße auf andere Art und Weise verändern. Es zeigen sich aber wiederkehrende Muster und Prozesse, die für den Wandel des Medienhandelns im Zuge der einzelnen Umbrüche typisch sind. Zur Dokumentation der empirischen Reichweite des Konzepts der mediatisierten Lebensführung soll an dieser Stelle nicht jeder einzelne Alltagsumbruch konkret analysiert werden (siehe ausführlich dazu Niemand, 2020, S. 117-215; Niemand, 2021), sondern ein abstrahierender Überblick über alle Umbrüche hinweg.

Entlang der Dimensionen der mediatisierten Lebensführung systematisiert zeigt sich eine beeindruckende Vielfalt von Antriebsfaktoren, die Dynamik im Medienrepertoire im Zuge von Transitionsprozessen auslösen können (siehe Abb. 2). Im Allgemeinen können die Antriebsfaktoren einerseits eine tiefere Alltagsintegration und somit einen Bedeutungsgewinn eines bestimmten Mediums anstoßen sowie andererseits zu einem Bedeutungsverlust führen. Der Bedeutungswandel eines Mediums findet dabei auf unterschiedlichen Ebenen statt:

- Ebene der Nutzungszeitpunkte und Nutzungsdauer,

- Ebene der räumlichen Medienarrangements,

- Ebene der sozialen Kontexte des Medienhandelns,

- Ebene der inhaltlichen Nutzung,

- Ebene des emotionalen Rezeptionserlebens,

- Ebene der sinnhaften Bedeutung bzw. des Nutzungsmotivs,

- Ebene der Medienausstattung und Nutzung bestimmter Medientechnologien. 
Abbildung 2: Antriebsfaktoren für Dynamik im Medienrepertoire: Gesamtübersicht

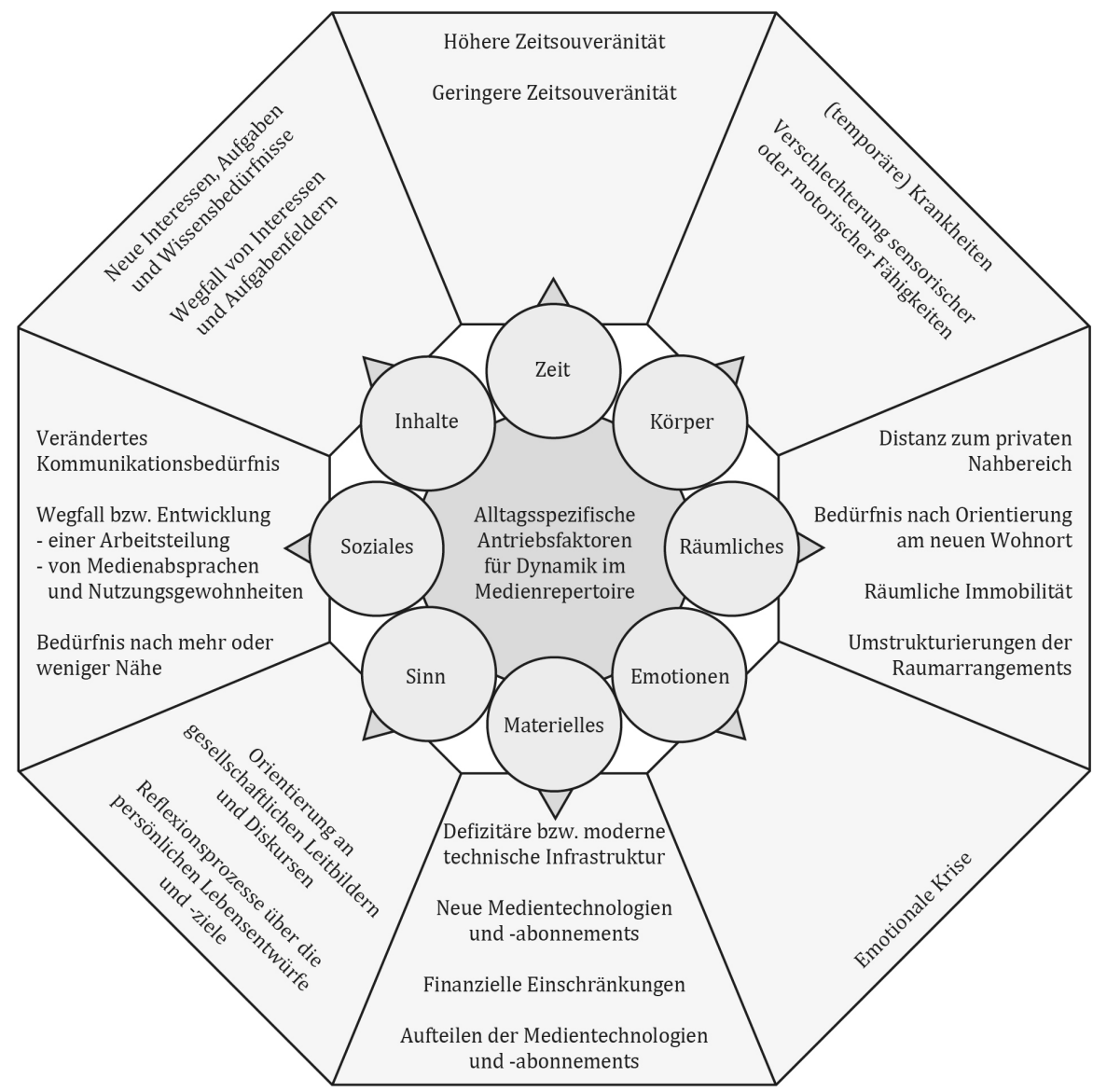

Quelle: Niemand, 2020, S. 237

Insgesamt verdeutlicht Abbildung 2, dass aus jeder Dimension der mediatisierten Lebensführung Dynamik entstehen kann. Wie vielschichtig die jeweiligen alltagsspezifischen Antriebsfaktoren als zentrale Rahmenbedingungen die sinnhafte Neugestaltung des Medienrepertoires prägen, wird im Folgenden entlang der Dimensionen aufgezeigt (siehe ausführlicher Niemand, 2020, S. 217-224).

\section{Zeit und Dynamik im Medienrepertoire}

Eine zentrale Ressource des Medienhandelns ist Zeit. Sowohl eine höhere Zeitsouveränität - zum Beispiel aufgrund veränderter Betreuungsaufgaben (Auszug eines Kindes) oder aufgrund des Wegfalls beruflicher Verpflichtungen (Eintritt in die Rente oder in den Mutterschutz) - als auch eine geringere Zeitsonveränität - zum Beispiel durch neue Betreuungsaufgaben (Elternschaft), Baumaßnahmen (Umzug) oder eine zusätzliche Ar- 
beitsbelastung (berufliche Veränderungen) - können einen Wandel der Mediennutzung anstoßen. „Dazugewonnene“ Zeit wurde von den Interviewten häufig verwendet, um Freizeitinteressen mit Medien zu verfolgen oder auch um „leere Zeit“ (Neverla, 1992, S. 167) zu füllen. Im Kontext knapp gewordener Zeitressourcen reduzierte sich hingegen in erster Linie die freizeitorientierte Mediennutzung, wobei Bewältigungsstrategien wie die Second-Screen-Nutzung oder die Nutzung von non-linearem Fernsehen dazu dienen sollten, die Auswirkungen der Zeitknappheit abzumildern.

\section{Inbaltliche Tätigkeiten und Dynamik im Medienrepertoire}

Nach einem Umbruch neu entstandene inhaltliche Interessen, Aufgaben und Wissensbedürfnisse führten bei einigen Paaren zu neuen Mediennutzungsmustern, wie Onlinerecherchen zum Hausbau (Umzug), Nutzung von Kommunikationsdiensten zur Ausübung des Berufs (berufliche Veränderungen), Rezeption von Elternzeitschriften und Onlineforen mit Bezug zur Elternschaft (Geburt eines Kindes) oder intensivem Lesen von Romanen per E-Book-Reader (Renteneintritt). Auch der Wegfall von Interessen und Aufgabenfeldern kann einen Wandel der Mediennutzung anstoßen, wenn zum Beispiel nach einem Renteneintritt die Notwendigkeit fehlt, für berufliche Zwecke online zu gehen, und so das Internet an Bedeutung verliert.

\section{Raum und Dynamik im Medienrepertoire}

Nach einigen Umbrüchen (Geburt eines Kindes, Umzug, Tod des Partners bzw. der Partnerin oder Auszug eines Kindes) stehen Funktionen der Wohnräume zur Disposition, sodass es zu erforderlichen oder auch erwünschten Umstrukturierungen der räumlichen Medienarrangements kommen kann. Zudem kann räumliche Immobilität durch ein Baby (Elternschaft) zur verstärkten Nutzung von Onlineshopping oder bei zuvor außerhäuslich aktiven Paaren zur vermehrten Fernsehrezeption am Abend führen. Um die räumliche Distanz zu den Kindern (Auszug eines Kindes und Trennung), zum Freundes- oder Bekanntenkreis (Umzug) oder zum neuen Partner bzw. zur neuen Partnerin bei einer Fernbeziehung (neue Partnerschaft) zu überwinden, werden Kommunikationsmedien wie Skype, WhatsApp oder das Telefon als Brücke zum sozialen Nahbereich genutzt und teils dafür gezielt angeeignet. Das Bedürfnis nach Orientierung am neuen Wobnort nach einem Ortswechsel (Umzug) kann durch Onlinerecherchen zu örtlichen Informationen sowie durch eine intensivere Nutzung von sozialen Medien oder von lokalen Medienangeboten befriedigt werden.

\section{Soziale Beziehungen und Dynamik im Medienrepertoire}

In der Dimension Soziales erweist sich die Beziehungskonstellation des Paares als wichtiger Faktor für die Ausgestaltung des Medienhandelns. Lösen sich Medienregeln, - $a b-$ sprachen und -gewohnheiten zwischen den Partnern auf (Trennung oder Tod des Partners/der Partnerin), können Medien selbstbestimmter entlang der eigenen Interessen genutzt werden. Der Autonomiegewinn führt nicht selten zu neuen Mediennutzungsmustern, wie beispielsweise zur Rezeption von Computerspielen, auf die zuvor aus Rücksichtnahme verzichtet wurde, oder zum Bedeutungsverlust des Fernsehabends, der zuvor vorwiegend dazu diente, Gemeinschaft als Paar herzustellen. Auch nene Medienregeln, -absprachen und-gewobnheiten (Geburt eines Kindes oder neue Partnerschaft) können durch den Verlust an Autonomie zu einem Wandel des Medienhandelns führen. Kommt es zu einem Wegfall der geschlechtsgebundenen Arbeitsteilung (Trennung oder 
Tod des Partners/der Partnerin) müssen zuvor aufgeteilte mediatisierte Praktiken (speziell mit dem Internet) nun selbst erledigt werden, wie Onlinebanking, die Wartung des Computers, die mediatisierte Kontaktpflege mit dem sozialen Umfeld oder der Kauf von Produkten im Internet. Die dafür notwendigen Kompetenzen müssen sich teils erst angeeignet werden. Eine neu ausgehandelte Arbeitsteilung (neue Partnerschaft oder Elternschaft) kann hingegen zu einer Entlastung und Abgabe mediatisierter Praktiken führen. Ferner führt ein erböhtes Kommunikationsbedürfnis zu einer veränderten Mediennutzung, wenn beispielsweise soziale Medien und Online-Partnerbörsen gehäuft genutzt werden, um neue Bekanntschaften zu schließen oder um alte zu intensivieren (Trennung), oder wenn Babyfotos mit Freundinnen und Freunden sowie mit der Familie geteilt werden (Elternschaft). Verändert sich das Bedürfnis nach Näbe und Distanz durch Beziehungskonflikte zwischen den Partnern (Trennung), kommt es zu einer zunehmend distanzierten und zumeist räumlich getrennten Mediennutzung.

\section{Emotionen und Dynamik im Medienrepertoire}

In dieser Dimension zeigen sich emotionale Krisen als Antriebsfaktor für Dynamik im Medienrepertoire. So kann nach einer Trennung oder auch einem als belastend erlebten Auszug eines Kindes eine intensive Medienrezeption dabei helfen, sich von dem Ereignis abzulenken. Eine eskapistische Mediennutzung stellt somit auch eine Art Bewältigungsstrategie der als krisenhaft erlebten Ereignisse dar.

\section{Sinnbezogene Orientierungen und Dynamik im Medienrepertoire}

Eine neuartige Orientierung an gesellschaftlichen Leitbildern und Diskursen kann Dynamik im Medienrepertoire anstoßen, wenn zum Beispiel medienpädagogische Diskurse im Medienhandeln wirksam werden (Geburt eines Kindes). Ferner können Reflexionsprozesse über die persönlichen Lebensentwürfe und -ziele zu einem Wandel der Mediennutzung führen, wenn beispielsweise über die intensive Nutzung von Onlinepartnerbörsen oder sozialen Medien versucht wird, den Lebensentwurf Partnerschaft und Familie zu realisieren (Trennung).

\section{Materielle Ressourcen und Dynamik im Medienrepertoire}

Finanzielle Einschränkungen (Hauskauf oder Trennung) können den Ausschlag geben, Investitionen in digitale Medien zurückzuhalten oder das Abonnement der Tageszeitung bzw. von abonnementbasierten Plattformen zu kündigen. Ferner kann die Verfügbarkeit neuer Medientechnologien und-abonnements (neue Partnerschaft oder Umzug) sowie die Aufteilung der Medienausstattung (Trennung) neue Nutzungsformen anstoßen. Zudem führte eine defizitäre technische Infrastruktur, bspw. nach einem Umzug in ländliche Regionen, zu Einschränkungen hinsichtlich einer datenintensiven Onlinenutzung, sodass bei einigen Paaren das Streamen von Videos, Filmen und Musik erheblich an Bedeutung verlor und durch eine vermehrte Fernsehrezeption ausgeglichen wurde. Kontrastierend nahm eine datenintensive Onlinenutzung zu, wenn nach einem Umzug in eine urbanere Region eine modernere technische Infrastruktur zur Verfügung stand.

\section{Körper und Dynamik im Medienrepertoire}

Dynamik im Medienrepertoire kann auch durch (temporäre) Krankbeiten angestoßen werden, bspw. wenn intensiv im Internet zu Symptomen und (alternativen) Behand- 
lungsmethoden recherchiert wird. Sind die für die Medienrezeption nötigen sensorischen oder motorischen Fähigkeiten eingeschränkt, kann dies zu einer Abkehr von bestimmten Mediendiensten führen. Wird eine Krankheit im Sinne von digitalem Stress mit der Nutzung von Medien in Verbindung gebracht, kann auch hier eine Beschränkung der eigenen Medienrezeption die Folge sein.

\subsection{Aushandlungsebenen der mediatisierten Lebensfübrung nach Umbrüchen}

Vor dem Hintergrund der oben skizzierten Alltagsveränderungen stellt sich die Frage, welche Faktoren für die Rezipierenden bei der Aushandlung der mediatisierten Lebensführung innerhalb der neuen Lebenssituation relevant sind. Wie entscheiden die Nutzer*innen, inwiefern Medien in ihrer neuen Lebenssituation sinnvoll sind? Während der Uses-and-Gratification-Approach die Zuwendung zu Medien ausschließlich über Bedürfnisse erklärt, zeigen unsere Analysen, dass eine solche Perspektive verkürzend ist. Vielmehr erfolgt die Aushandlung innerhalb eines komplexen Zusammenspiels aus lebenssituationsspezifischen Entfaltungschancen, Zwängen und Erwartungen sowie Bedürfnissen und Daseinsthemen (siehe Abb. 1 sowie theoretische Erörterung in Abschnitt 4). Dies soll folgend anhand ausgewählter Befunde näher erläutert werden.

\section{Beispiel Umzug}

Die Paare, die in eine ländlichere Region umgezogen waren, reduzierten aufgrund einer spürbaren Verschlechterung der Internetverbindung ihre Onlinenutzung drastisch. Obwohl die Interviewten das Bedürfnis hatten, ihre zuvor etablierte Internetnutzung auch in der neuen Wohngegend aufrechtzuerhalten, konnten sie dies aufgrund der eingeschränkten Entfaltungschancen durch die defizitäre technische Infrastruktur nicht umsetzen. Unter diesen Umständen wurde das zuvor vernachlässigte Fernsehen wieder sinnvoll, um alternativ zum Internet die Bedürfnisse der Interviewten zu befriedigen. Aus einer kritischen Perspektive zeigen sich hier Ungleichheiten entlang der Differenzlinie Stadt-Land.

\section{Beispiel Tod des Partners}

Die Interviewte, deren Partner verstarb, sah sich vor dem Tod ihres Mannes mit seiner Erwartungshaltung konfrontiert, im Beisein ihres Mannes kein Radio und keine Musik zu hören - obwohl sie das Bedürfnis danach hatte. Die Auflösung dieser Reglementierung durch den Tod - so makaber dies auch klingen mag - bedeutete für sie neue Entfaltungschancen, weil sie nun selbstbestimmter Radio und Musik hören konnte (was sie auch ausgiebig tat). Das Radio wurde unter den veränderten Rahmenbedingungen für sie also sinnhaft.

\section{Beispiel Elternschaft}

Die Paare, die Eltern wurden, sahen sich dem Zwang ausgesetzt, den Tagesrhythmus vorwiegend an den Bedürfnissen des Kindes auszurichten. Zudem wurden medienpädagogische Diskurse (wie keine Fernsehnutzung im Beisein des Kindes) als Erwartungsbaltung an die Elternrolle im Medienhandeln der Paare wirksam. Beides stand in $\mathrm{Zu}-$ sammenhang mit einer Reduzierung der freizeitorientierten Mediennutzung - obwohl das Bedürfnis danach prinzipiell noch vorhanden war. Unter den veränderten Rahmenbedingungen verloren freizeitorientierte Medien also ihre Sinnhaftigkeit. Gleichzeitig 
entwickelte sich mit der Geburt des Kindes ein neues Daseinsthema, das zum Beispiel in Form von neuen Wissens- und Kompetenzbedürfnissen zur Fürsorge des Kindes die Mediennutzung prägte. Insgesamt zeigen die Analysen zur Elternschaft, dass sich das Medienhandeln der Mütter stärker als das der Väter veränderte, weil die Mütter mehr Verantwortung für Erziehung und Fürsorge übernahmen. In Zusammenhang mit solchen Retraditionalisierungstendenzen, die durch die Analyse der Medienpraktiken im Zuge eines Alltagsumbruchs sichtbar werden, lassen sich auch kritische Fragen zu Geschlechterverhältnissen stellen.

Die Befunde der empirischen Studie lassen sich folglich entlang dieses Analyserasters gewinnbringend erörtern. Grundsätzlich kann die Neustrukturierung der mediatisierten Lebensführung zielgerichtet und reflexiv erfolgen, muss es aber nicht. Mit Blick auf Geschlechterverhältnisse oder hinsichtlich der Ungleichheiten zwischen Stadt und Land wird zudem angedeutet, dass sich dieses Raster auch zur Analyse von Machtkonstellationen eignet. Es ist in vielfacher Hinsicht anschlussfähig für kritische Perspektiven und erlaubt - obwohl dies hier nicht im Fokus stand - anhand der konkreten Medienpraktiken der Menschen systematisch Rückschlüsse auf gesamtgesellschaftliche Verhältnisse, z. B. durch intersektionale Analysen entlang zentraler Differenzkategorien wie Geschlecht, Klasse, Ethnie, Alter, sexuelle Orientierung, Stadt-Land, Religion oder auch Körper (siehe z. B. bezogen auf die Internetnutzung Carstensen \& Winker, 2012).

\section{Resümee: Mediatisierte Lebensführung, Alltagsumbrüche und ihre Relevanz für die Rezeptionsforschung}

In diesem Artikel wurde das Konzept der mediatisierten Lebensführung entfaltet, das sich speziell dazu eignet, um den Wandel des Medienhandelns im Zuge von Alltagsumbrüchen zu analysieren. Die empirische Reichweite des Konzepts wurde anhand der Befunde einer qualitativen Panelstudie veranschaulicht. Mit Blick auf den Mehrwert für die kommunikationswissenschaftliche Rezeptionsforschung lassen sich speziell drei Aspekte betonen: Erstens werden Alltagsumbrüche als relevante Analysekategorie in das Sichtfeld der Rezeptionsforschung gerückt. Gerade in „modernen“ Gesellschaften, in denen die Lebensführung „offen, entscheidungsabhängig und als Aufgabe in das Handeln jedes einzelnen gelegt wird“ (Beck, 1986, S. 216), erweisen sich Alltagumbrüche als vielversprechender Zugang, um über die Identifikation von alltagsspezifischen Antriebskräften zu erhellen, warum sich die Mediennutzung im Laufe des Lebens verändert.

Als besonders instruktiv für die Analyse hat sich die Systematisierung der mediatisierten Lebensführung in die Strukturdimensionen zeitlich, räumlich, inhaltlich, sozial, sinnbezogen, materiell, emotional und körperlich erwiesen. So konnten Veränderungen in der Alltagsstruktur zielgenau und systematisch mit einem Wandel des Medienhandels in Beziehung gesetzt und zugleich gezeigt werden, dass Dynamik im Medienrepertoire auf vielschichtige Weise durch unterschiedliche Kontextfaktoren angestoßen wird. Ferner können durch den präzisen Blick auf die Alltagsveränderungen homogenisierende Beschreibungen der Mediennutzung von einzelnen Milieus (wie die Konservativen), sozialer Gruppen (wie die Migrant*innen) oder einzelner Lebensphasen (wie in der Kindheit) vermieden werden. Vielmehr lässt sich so ein tieferes Verständnis entwickeln, warum welche Medien in spezifischen Lebenssituationen bedeutsam sind. Das kommunikative Handeln mit Medien ändert sich eben nicht, weil ein Mensch „konservativ“ oder „Migrant“ wird oder weil ein Mensch von der „Lebensphase Kindheit“ in die „Lebensphase Jugend“ eingetreten ist, sondern weil spezifische Anforderungen innerhalb der einzelnen Lebensbereiche neu an den Menschen herangetragen werden. 
Dies leitet zum zweiten Aspekt über, der Einsicht, dass Medien in einem komplexen Spannungsfeld aus lebenssituationsspezifischen Entfaltungschancen, Zwängen und Erwartungen sowie Bedürfnissen und Daseinsthemen angeeignet werden und so ihren Sinn entfalten. Die Rahmenbedingungen in diesem Spannungsfeld ändern sich im Verlauf eines Übergangs - z. B. durch die Veränderung von Zeitsouveränität (zeitlich), die Auflösung einer geschlechtsspezifischen Arbeitsteilung (sozial) oder den Wandel der Infrastruktur (materiell) - und stoßen somit Dynamik im Medienrepertoire an. Denn Umbrüche wie ein Berufsbeginn, die Geburt eines Kindes oder auch der Beginn der Rente prägen, in welchen „Handlungsfeldern und Sozialwelten“ (Krotz, 2014, S. 14) sich ein Mensch in seinem Alltag bewegt, welche Daseinsthemen er dabei zu bewältigen hat, welche Bedürfnisse ihn antreiben, über welche Ressourcen er verfügt sowie welche Zwänge und Erwartungen auf ihn einwirken, und all dies hat einen maßgeblichen Einfluss darauf, wie er mit Medien kommunikativ handelt.

Insgesamt stellt diese Heuristik eine neue Systematik bereit, um Medienaneignung $\mathrm{zu}$ analysieren. Während beispielsweise der Uses-and-Gratification-Approach die Zuwendung zu Medien zumeist mittels situationsübergreifender und individueller Nutzungsmotive wie Orientierung, Unterhaltung oder Information erklären möchte (siehe u. a. zur Kritik daran Schweiger, 2007, S. 66-73), erlaubt diese Systematik eine komplexere Betrachtungsweise. Denn es kann herausgearbeitet werden, inwiefern (mediale) Bedürfnisse überhaupt erst aus der Lebensführung heraus entstehen und warum Menschen nicht immer ihren (medialen) Bedürfnissen folgen (können), da diese im Spannungsfeld mit den anderen Faktoren stehen. Ein besonders interessanter Fokus ist hierbei, inwiefern Medien bei der Bewältigung von Alltagsumbrüchen innerhalb der verschiedenen Lebensbereiche und Handlungsfelder unterstützen (und wo Potenzial wäre, die Bewältigung mit Hilfe von Medien weiter zu fördern).

Schließlich bietet das Konzept der mediatisierten Lebensführung drittens ein Ordnungsschema, um zu konkretisieren, welche spezifischen Kontextbereiche bei der Analyse der Medienaneignung im Alltag fokussiert werden, denn aufgrund der Größe des Konstrukts Alltag kann es durchaus sinnvoll sein, sich auf einzelne Teilbereiche und Handlungsfelder der mediatisierten Lebensführung zu reduzieren (und damit gleichzeitig anzuzeigen, welche Bereiche nicht erforscht werden). So kann die Bedeutung von Medien z. B. gezielt innerhalb einzelner Beziehungsphasen, im Rahmen der Organisation von Freundschaftsbeziehungen, mit Blick auf die politische Teilhabe oder auch hinsichtlich der Interaktion einzelner Lebensbereiche untersucht werden, wenn beispielsweise danach gefragt wird, wie Berufs- und Familienleben im Kontext von Home Office ausbalanciert werden.

\section{Literatur}

Augustin, E. (2015). BlogLife. Zur Bewältigung von Lebensereignissen in Weblogs. Transcript.

Bachmann, G., \& Wittel, A. (2006). Medienethnographie. In R. Ayaß \& J. Bergmann (Hrsg.), Qualitative Methoden der Medienforschung (S. 183-219). Rowohlt.

Beck, U. (1986). Risikogesellschaft: auf dem Weg in eine andere Moderne. Suhrkamp.

Beck, U. (1996). Das Zeitalter der Nebenfolgen und die Politisierung der Moderne. In U. Beck, A. Giddens \& S. Lash (Hrsg.), Reflexive Modernisierung. Eine Kontroverse (S. 19-112). Suhrkamp.

Berger, J., \& Schönberger, K. (2016). Zur Rolle von Medien bei der Bewältigung neuer Lebensaufgaben am Anfang der Pensionszeit. kommunikation.medien, 6, 1-26.

Bergius, R. (2009). Bedürfnis. In H. O. Häcker \& K. H. Stapf (Hrsg.), Dorsch Psychologisches Wörterbuch (S. 114-115). Huber.

Carstensen, T., \& Winker, G. (2012). Intersektionalität in der Internetforschung. MEK Medien $\mathcal{E}$ Kommunikationswissenschaft, 60(1), 3-23. 
Charlton, M., \& Neumann-Braun, K. (1992). Medienkindheit - Medienjugend. Eine Einführung in die aktuelle kommunikationswissenschaftliche Forschung. Quintessenz.

Couldry, N., \& Hepp, A. (2017). The mediated construction of reality. Polity.

Eimeren, B. van, Gaber, R., Koch, W., \& Schröter, C. (2017). Mediennutzung junger Berufseinsteiger. Ergebnisse einer qualitativen Untersuchung. Media Perspektiven, 1, 16-25.

Gauntlett, D., \& Hill, A. (1999). TV Living: Television, culture and everyday life. Routledge.

Giddens, A. (1996). Leben in einer posttraditionalen Gesellschaft. In U. Beck, A. Giddens \& S. Lash (Hrsg.), Reflexive Modernisierung. Eine Kontroverse (S. 113-194). Suhrkamp.

Hackl, C. (2001). Fernsehen im Lebenslauf. Eine medienbiographische Studie. UVK.

Hartmann, M. (2013). Domestizierung. Nomos.

Hasebrink, U., \& Domeyer, H. (2012). Media repertoires as patterns of behaviour and as meaningful practices: a multimethod approach to media use in converging media environments. Participations Journal of Audience E Reception Studies, 9(2), 757-779.

Jurczyk, K., \& Rerrich, M. (1993). Einführung: Alltägliche Lebensführung, der Ort, wo „alles zusammenkommt“. In K. Jurczyk \& M. Rerrich (Hrsg.), Die Arbeit des Alltags. Beiträge zu einer Soziologie der alltäglichen Lebensfübrung (S. 11-45). Lambertus.

Karnowski, V. (2003). Von den Simpsons zur Rundschau. Wie sich Fernsehnutzung im Laufe des Lebens verändert. Angewandte Medienforschung. Schriftenreihe des Medien Instituts Ludwigshafen, (Vol. 28). Reinhard Fischer.

Kohli, M. (1985). Die Institutionalisierung des Lebenslaufs: Historische Befunde und theoretische Argumente. Kolner Zeitschrift für Soziologie und Sozialpsychologie, 37, 1-29.

Krotz, F. (2001). Die Mediatisierung kommunikativen Handelns. Der Wandel von Alltag und sozialen Beziehungen, Kultur und Gesellschaft durch die Medien. Westdeutscher Verlag.

Krotz, F. (2007). Mediatisierung. Fallstudien zum Wandel von Kommunikation. Springer VS.

Krotz, F. (2012). Mediatisierung als Meta-Prozess. In J. Hagenah \& H. Meulemann (Hrsg.), Mediatisierung der Gesellschaft? (S. 19-41). LIT Verlag.

Krotz, F. (2014). Einleitung: Projektübergreifende Konzepte und theoretische Bezüge der Untersuchung mediatisierter Welten. In F. Krotz, C. Despotovic \& M. Kruse (Hrsg.), Die Mediatisierung sozialer Welten (S. 7-32). Springer VS.

Krotz, F. (2015). Medienwandel in der Perspektive der Mediatisierungsforschung: Annäherung an ein Konzept. In S. Kinnebrock, C. Schwarzenegger \& T. Birkner (Hrsg.), Theorien des Medienwandels (S. 119-140). Halem.

Krotz, F., \& Thomas, T. (2007). Domestizierung, Alltag, Mediatisierung: Ein Ansatz zu einer theoriegerichteten Verständigung. In J. Röser (Hrsg.), MedienAlltag. Domestizierungsprozesse alter und neuer Medien (S. 31-42). VS Verlag für Sozialwissenschaften.

Livingstone, S. (2015). From Mass to Social Media? Advancing Accounts of Social Change. Social Media + Society, 1-3.

Lundby, Knut (2009). Mediatization. Concept, changes, consequences. New York: Peter Lang.

Meyrowitz, J. (1995): Medium Theory. In D. Crowley \& D. Mitchell (Hrsg.), Communication Theory Today (S. 50-77). Polity Press.

Neumann-Braun, K. (2005). Strukturanalytische Rezeptionsforschung. In L. Mikos \& C. Wegener (Hrsg.), Qualitative Medienforschung: Ein Handbuch (S. 58-66). UVK.

Neverla, I. (1992). Fernseh-Zeit. Zuschauer zwischen Zeitkalkül und Zeitvertreib. Eine Untersuchung zur Fernsehnutzung. Ölschläger.

Niemand, S. (2020). Alltagsumbrüche und Medienhandeln. Eine qualitative Panelstudie zum Wandel der Mediennutzung in Übergangsphasen. Springer VS.

Niemand, S. (2021). Elternschaft und Dynamik im Medienrepertoire. Wie und warum junge Eltern nach der Geburt eines Kindes ihre Mediennutzung anpassen. Merz, 65(3), 79-85.

Prommer, E. (2009). Mediengenerationen - Kinobesuch im Lebenslauf. In B. Schorb, A. Hartung \& W. Reißmann (Hrsg.), Medien und höheres Lebensalter. Theorie - Forschung - Praxis (S. 259264). VS Verlag für Sozialwissenschaften.

Röser, J. (2007). Der Domestizierungsansatz und seine Potenziale zur Analyse alltäglichen Medienhandelns. In J. Röser (Hrsg.), MedienAlltag. Domestizierungsprozesse alter und neuer Medien (S. 15-30). VS Verlag für Sozialwissenschaften. 
Röser, J. (2016). Nicht standardisierte Methoden in der Medienrezeptionsforschung. In S. Averbeck-Lietz \& M. Meyen (Hrsg.), Handbuch nicht standardisierte Methoden in der Kommunikationswissenschaft (S. 481-497). Springer VS.

Röser, J., \& Müller, K. F. (2017). Der Domestizierungsansatz. In L. Mikos \& C. Wegener (Hrsg.), Qualitative Medienforschung. Ein Handbuch (S. 156-163). UVK.

Röser, J., Müller, K. F., Niemand, S., \& Roth, U. (2017). Die Domestizierung des Internets zwischen Dynamik und Beharrung: Häusliches Medienhandeln 2008-2016. In F. Krotz, C. Despotović $\&$ M. Kruse (Hrsg.), Mediatisierung als Metaprozess. Transformationen, Formen der Entwicklung und die Generierung von Neuem (S. 139-162). Springer VS.

Röser, J., Müller, K. F., Niemand, S., Peil, C., \& Roth, U. (2018). Medienethnografische Porträts als Auswertungsinstrument: Techniken der kontextsensiblen Rezeptionsanalyse. In A. Scheu (Hrsg.), Auswertung qualitativer Daten in der Kommunikationswissenschaft (S. 193-207). Springer VS.

Röser, J., Müller, K. F., Niemand, S., Peil, C., \& Roth, U. (2019). Das mediatisierte Zubause im Wandel. Eine qualitative Panelstudie zur Verbäuslichung des Internets. Springer VS.

Roth, U., \& Röser, J. (2019). Domestizierungsansatz: Medienaneignung und Geschlechterordnungen. In J. Dorer, B. Geiger, B. Hipfl \& V. Ratković (Hrsg.), Handbuch Medien und Geschlecht. Perspektiven und Befunde der feministischen Kommunikations- und Medienforschung (online first). Springer VS. https://doi.org/10.1007/978-3-658-20712-0_21-1.

Schweiger, W. (2007). Theorien der Mediennutzung. Eine Einführung. Springer VS.

Sennett, R. (1998). Der flexible Mensch. Die Kultur des neuen Kapitalismus. Berlin-Verlag.

Silverstone, R. (1994). Television and everyday life. Routledge.

Silverstone, R., \& Haddon, L. (1996). Design and the domestication of information and communication technologies. Technical change and everyday life. In R. Silverstone \& R. Mansell (Hrsg.), Communication by design. The politics of information and communication technologies (S. 44-74). Oxford University Press.

Silverstone, R., Hirsch, E., \& Morley, D. (1992). Information and communication technologies and the moral economy of the household. In R. Silverstone \& E. Hirsch (Hrsg.), Consuming technologies. Media and information in domestic spaces (S. 15-31). Routledge.

Steinmaurer, T. (2013). Kommunikative Dauervernetzung. Historische Entwicklungslinien und aktuelle Phänomene eines neuen Dispositivs. Medien Journal, 4, 4-17.

Thomae, H. (1988). Das Individuum und seine Welt. Eine Persönlichkeitstheorie. Hogrefe.

Vorderer, P. (2015). Der mediatisierte Lebenswandel. Permanently online, permanently connected. Publizistik, 60, 259-276.

Voß, G. (1991). Lebensführung als Arbeit. Über die Autonomie der Person im Alltag der Gesellschaft. Enke.

Voß, G. (1995). Entwicklung und Eckpunkte des theoretischen Konzepts. In Projektgruppe Alltägliche Lebensführung (Hrsg.), Alltägliche Lebensfübrung. Arrangements zwischen Traditionalität und Modernisierung (S. 23-43). VS Verlag für Sozialwissenschaft.

Voß, G. (2001). Der eigene und der fremde Alltag. In G. Voß \& M. Weihrich (Hrsg.), tagaus tagein. Neue Beiträge zur Soziologie Alltäglicher Lebensfübrung (S. 203-217). Rainer Hampp.

Welzer, H. (1990). Zwischen den Stüblen. Eine Längsschnittuntersuchung zum Übergangsprozess von Hochschulabsolventen. Deutscher Studien-Verlag.

Welzer, H. (1993a). Transitionen. Zur Sozialpsychologie biographischer Wandlungsprozesse. edition diskord.

Welzer, H. (1993b). Transitionen - ein Konzept zur Erforschung biographischen Wandels. handlung - kultur - interpretation, Zeitschrift für Sozial- und Kulturwissenschaften 2(3), 137-154. 